\title{
On the dynamics and linear stability of one-dimensional steady detonation waves
}

\author{
Filipe Carvalhoł and Ana Jacinta Soares \\ Centro de Matemática, Universidade do Minho, Braga, Portugal \\ E-mail: filipecarvalho@esce.ipvc.pt, ajsoares@math.uminho.pt
}

\begin{abstract}
A detailed analysis of the dynamics and linear stability of a steady one-dimensional detonation wave propagating in a binary reactive system with an Arrhenius chemical kinetics of type $A+A \rightleftharpoons B+B$ is carried out. Starting from the frame of the kinetic theory, the binary reactive mixture is modelled at the mesoscopic scale by the reactive Boltzmann equation (BE), assuming hard sphere cross sections for elastic collisions and step cross sections with activation energy for reactive interactions. The corresponding hydrodynamic limit is based on a second-order non-equilibrium solution of the BE obtained in a previous paper, using the Chapman-Enskog method in a chemical regime for which the reactive interactions are less frequent than the elastic collisions. The resulting hydrodynamic governing equations are the reactive Euler equations, including a rate law which exhibits an explicit dependence on the reaction heat and forward activation energy of the chemical reaction. These equations are used to describe the spatial structure of the steady detonation wave solution and investigate how this structure varies with the reaction heat. The response of the steady solution to one-dimensional disturbances is studied using a normal mode linear approach which leads to an initial value problem for the state variable disturbances in the reaction zone. The stability problem is treated numerically, using an iterative shooting technique to determine the unstable modes. The analysis here developed emphasizes the influence of the chemical reaction heat and activation energy on the linear stability spectra.
\end{abstract}

PACS numbers: 51.10.+y, 47.45.Ab 47.70.Pq, 82.33.Vx, 47.20.-k

Keywords: Kinetic theory. Boltzmann equation. Chemical reactions. Reactive flows. Steady detonation waves. Hydrodynamic stability.

Accepted (May 2, 2012) for publication in J. Phys. A: Math. Theor.

‡ Institutional address: Departamento de Ciências Exactas, Instituto Politécnico de Viana do Castelo, Portugal 


\section{Introduction}

Kinetic approaches to chemically reactive fluids and direct simulations of Monte Carlo method have been widely used in literature, with the aim of studying fluid dynamical applications in which the chemical reaction plays a crucial role $[1,2,3,4,5,6,7,8,9$, $10,11,12]$. Starting from a kinetic approach, a consistent macroscopic picture can be obtained in terms of the corrected balance equations that include the non-equilibrium effects due to the chemical reaction. In particular, in view of practical applications dealing with reactive fluids, the kinetic approach can help to understand and describe the chemical reaction that plays a crucial role in the process. On the other hand, the propagation of detonation waves in gases is a problem of great practical importance, due to the related engineering applications, such as propulsion devices, power generation, safety and military issues, explosive welding, hazard prevention and hard rock mining, among others.

Motivated by this background, the main purpose of the present paper is to study the propagation and linear stability of steady detonation waves, analyzing the macroscopic reactive balance laws derived from a simple kinetic modelling of a binary reactive mixture. Starting from the Boltzmann equation for the gaseous mixture with bimolecular chemical reaction of symmetric type, a closure procedure of the balance laws is performed in a chemical regime for which the reactive interactions are less frequent than the elastic collisions. The resulting hydrodynamic equations include some non-equilibrium corrections, namely those of the reaction rate due to the heat of the chemical reaction. The approach permits to perform an interesting analysis of the stability problem with emphasis on the influence of the reaction heat on the stability spectrum.

There exists a rather vast bibliography on the detonation subject, covering either mathematical and physical modelling approaches or numerical and experimental studies. The interested reader is addressed to the papers $[9,12,13,14,15,16,17,18,19,20]$, for example, and to the books $[21,22,23,24]$ for an exhaustive account of the literature on this subject. Concerning the investigation of the detonation problem within kinetic theory, the existing literature covers essentially the kinetic modelling of the one-dimensional steady propagating wave, and give some contributions at the level of analytical predictions and numerical simulations [4, 9, 12, 19].

Most widely used detonation models include the Chapman-Jouguet and the Zeldovich, von Neumann and Doering (ZND) idealized theories [21, 22], the WoodKirkwood non-ideal theory $[25,26,27]$ as well as the detonation shock dynamics asymptotic theory [28]. In particular, the ZND theory gives a good and accepted description of the dynamics of a steady one-dimensional planar detonation wave in a gaseous explosive mixture, with a single-step Arrhenius rate law for an exothermic chemical reaction. The structure of the detonation wave consists of an inert shock followed by a finite reaction zone where the chemical process takes place. The flow is one-dimensional and steady in the shock attached frame. The mathematical analog is 
the hyperbolic set of the reactive Euler equations and the related Rankine-Hugoniot conditions.

Complexities in the reactive system, such as an endothermic chemical reaction, a mole change reaction, two chemical reactions with one being reversible, transport effects or another dissipative process, can produce the so-called pathological-type detonation [22]. Typically, the system involves two consecutive chemical reactions with the first one being exothermic and the second one endothermic. There exist some examples of pathological detonations in astrophysics, related to nuclear detonation waves in the stellar material of white dwarf stars $[29,30]$.

Experimental and computational studies show that the detonation tends to be structurally unstable and that the instabilities propagate in a quasi-periodic oscillating manner $[21,23]$. The reaction zone behind the shock is extremely sensitive to small rear boundary perturbations and, as a result, the detonation wave presents, in general, an unstable configuration.

The dynamics of such propagating instabilities can provide useful information about the unsteady structure and elucidate about the detonation mechanism. The usual first step is a hydrodynamic stability analysis of the steady detonation solution. Assuming that the perturbations are small, a linearized theory is used to describe their evolution and determine the instability modes. The results of this linear analysis are relevant for detonation waves in channels or square tubes and give important information about the growth rate of the instabilities and the influence of the detonation parameters in the instability behaviour [13, 31].

An extensive and valuable study of the linear stability problem was developed by Erpenbeck using a Laplace transform approach and a numerical technique based on the Nyquist-winding theorem to determine the number of unstable solutions $[32,33]$. The works by Abouseif and Toong [13, 14], Buckmaster, Ludford and Neves [34, 35] and Majda an Rosales [36] gave important analytical and numerical contributions for the physical interpretations of the instability behaviour. A further relevant contribution to the linear detonation stability was given by Lee and Stewart in paper [37], using a normal mode approach and a numerical shooting technique to find the unstable modes. An extensive literature based on similar approaches and using rather sophisticated numerical techniques, include, among others, the papers $[17,18,38,39,40,41,42,43,44,45,46,47]$ for ZND detonation, and [17, 29, 30, 48] for pathologic-type detonations. Modern computer facilities allowed to produce several experimental and computational studies, as for example those of papers $[15,16,49,50]$.

Although a linear stability analysis can describe some relevant physical effects of the perturbations [13], it requires that the steady character of the detonation wave be only slightly perturbed and therefore its validity is restricted to the initial stage of very small amplitude oscillations. A non-linear stability analysis is needed for detonation waves whose structure is very far from that of the steady wave. A typical case is the curved detonation wave propagating through an unconfined material, for which more real effects of multi-dimensional instabilities are observed, such as bifurcations to multi- 
mode and irregular oscillations, and "diamon" or "fish scale" patterns are produced $[23,31,51]$.

Concerning the detonation stability analysis in the context of the kinetic theory for chemically reacting gases, the hydrodynamic linear stability of steady detonation waves has been investigated for the first time in paper [10]. The emphasis of paper [10] is on the mathematical formulation and solutions to the stability problem in the kinetic frame, for a quaternary gas mixture with a reversible bimolecular chemical reaction. Some numerical results and visualizations are shown regarding the time evolution of the eigenfunctions for both instability and stability pictures as well as at conditions of neutral stability. However, the considered kinetic modelling does not include the effects of the reaction heat neither those of the activation energy, so that the stability picture remains incomplete. Starting from the kinetic formulation proposed in [10], the steady detonation solution characterized in paper [19] is revisited here, with the aim of investigating its linear stability. The main objective is to develop a detailed hydrodynamic stability analysis, and investigate the influence of the reaction heat and activation energy on the stability behaviour. A first contribution in this direction was presented in paper [20], where some numerical simulations have been shown about the structure of the detonation wave and its linear stability. This preliminary stability analysis is expanded and detailed in the present paper, exploiting the non-equilibrium effects due to the heat of the chemical reaction. Both the mathematical formulation of the stability problem and the numerical method of solution are explained in detail. Some numerical simulations are performed and the results are presented and discussed.

The paper is organized as follows. The governing equations of the reactive flow are presented in section 2, where the relevant chemical kinetics properties are summarized and the macroscopic analog for the detonation problem and related stability analysis is explained. In section 3, the steady state one-dimensional solutions are obtained and interpreted. The normal mode stability analysis is treated in section 4, where the stability problem for the eigenfunctions and growth rate perturbation is deduced, and then the numerical technique used in the simulations is described. In section 5, some representative computational results for the stability behaviour in the parameter space are given and discussed. Finally, the conclusions and some closing remarks, together with some ideas for future work, are presented in section 6 .

\section{Governing equations of the reactive flow}

The detonating gaseous mixture considered in this paper is an idealized system described by the reactive Boltzmann equation proposed in [6] for a binary gaseous mixture undergoing a single reversible chemical reaction of symmetric type. The model reactive system is based on some simplifying assumptions and the theoretical treatment of the nonequilibrium effects is focused both on the deviations caused by the chemical reaction and on the influence of the reaction heat. The mathematical and chemical features of the kinetic modelling are briefly summarized in the present section, in view of describing 
steady detonation wave solutions and studying their linear stability.

\subsection{Chemical kinetics framework}

Consider a binary gaseous mixture whose constituents, A and B, have equal molecular mass $m$ and binding energies $E_{A}$ and $E_{B}$. Vibrational and rotational molecular degrees of freedom are not taken into account. The gas particles can undergo binary elastic collisions as well as collisions with chemical reaction according to the single reversible symmetric law $\mathrm{A}+\mathrm{A} \rightleftharpoons \mathrm{B}+\mathrm{B}$. At the mesoscopic scale, the thermodynamical behaviour of the mixture is modelled by the following system of Boltzmann equations for the constituent distribution functions $f_{\alpha}\left(\boldsymbol{x}, \boldsymbol{c}_{\alpha}, t\right)$, with $\boldsymbol{x}, \boldsymbol{c}_{\alpha} \in \mathbb{R}^{3}$ and $t \in \mathbb{R}^{+}$,

$$
\frac{\partial f_{\alpha}}{\partial t}+\sum_{i=1}^{3} \boldsymbol{c}_{i}^{\alpha} \frac{\partial f_{\alpha}}{\partial \boldsymbol{x}_{i}}=\mathcal{Q}_{\alpha}^{E}+\mathcal{Q}_{\alpha}^{R}, \quad \alpha=A, B,
$$

where $\mathcal{Q}_{\alpha}^{E}$ and $\mathcal{Q}_{\alpha}^{R}$ are the elastic and reactive collision terms, respectively, given by

$$
\begin{aligned}
\mathcal{Q}_{\alpha}^{E} & =\sum_{\beta=A}^{B} \int\left(f_{\alpha}^{\prime} f_{\beta}^{\prime}-f_{\alpha} f_{\beta}\right) d^{2}\left(g_{\beta \alpha} \cdot \mathbf{k}_{\beta \alpha}\right) d \mathbf{k}_{\beta \alpha} d \mathbf{c}_{\beta}, \\
\mathcal{Q}_{\alpha}^{R} & =\int\left[f_{\beta} f_{\beta}^{\prime}-f_{\alpha} f_{\alpha}^{\prime}\right] \sigma_{\alpha}^{\star}\left(g_{\alpha} \cdot \mathbf{k}_{\alpha}\right) d \mathbf{k}_{\alpha} d \mathbf{c}^{\prime}{ }_{\alpha} .
\end{aligned}
$$

In expression (2), the primes denote post collisional distribution functions, $\mathbf{d}$ the elastic particle diameter, $g_{\beta \alpha}$ the relative velocity between the $\alpha$ and $\beta$ particles, $\mathbf{k}_{\beta \alpha}$ the unit collision vector and $d \mathbf{k}_{\beta \alpha}$ the element of solid angle for elastic collisions. In expression (3), the primes are used to distinguish two identical particles that participate in the reactive event, $\mathbf{k}_{\alpha}$ is the unit collision vector, $d \mathbf{k}_{\alpha}$ the element of solid angle for reactive collisions, and the term $\sigma_{\alpha}^{\star}$ is the differential reactive step cross section,

$$
\sigma_{\alpha}^{\star}=\mathrm{d}_{r}^{2} \quad \text { for } \quad \gamma_{\alpha}>\varepsilon_{\alpha}^{\star} \quad \text { and } \quad \sigma_{\alpha}^{\star}=0 \text { for } \gamma_{\alpha}<\varepsilon_{\alpha}^{\star},
$$

where $\mathrm{d}_{r}$ is reactive diameter $\left(\mathrm{d}_{r}=s \mathrm{~d}\right.$, with $s$ being the steric factor $), \gamma_{\alpha}$ the relative translational energy, and $\varepsilon_{\alpha}^{\star}$ the forward $(\alpha=A)$ and backward $(\alpha=B)$ activation energy. Parameters $\gamma_{\alpha}$ and $\varepsilon_{\alpha}^{\star}$ are expressed in units of the mixture thermal energy, $k T$, with $k$ being the Boltzmann constant and $T$ the temperature of the mixture,

$$
\gamma_{\alpha}=\frac{m g_{\alpha}^{2}}{4 k T}, \quad \varepsilon_{A}^{\star}=\frac{\varepsilon_{A}}{k T}, \quad \varepsilon_{B}^{\star}=\varepsilon_{A}^{\star}-Q_{R}^{\star}, \quad Q_{R}^{\star}=\frac{Q_{R}}{k T},
$$

where $Q_{R}=2\left(E_{B}-E_{A}\right)$ is the reaction heat, which is identified as the difference between the total kinetic energies of the reactants and products, $Q_{R}=\frac{1}{4} m\left(g_{A}^{2}-g_{B}^{2}\right)$.

As stated in paper [6], the collision terms (2) and (3) are consistent with conservation laws of mass, momentum and total energy as well as with the entropy inequality. They assure the correct chemical exchanges and reproduce the expected form of the mass action law. Equations (1-3) describe the mesoscopic state of the reactive mixture and their solution, $f_{\alpha}$, accommodates the non-equilibrium effects considered in the present modelling, in particular those due to the presence of the chemical reaction and those caused by elastic interactions and free streaming. 


\subsection{Macroscopic analog}

At the macroscopic scale, the thermodynamical state of the mixture is specified by the number densities $n_{\alpha}$ of the constituents, and by the mean velocity $v_{i}$ and temperature $T$ of the mixture,

$n_{\alpha}=\int f_{\alpha} d \boldsymbol{c}_{\alpha}, \quad v_{i}=\frac{1}{n} \sum_{\alpha=A}^{B} \int \boldsymbol{c}_{i}^{\alpha} f_{\alpha} d \boldsymbol{c}_{\alpha}, \quad T=\frac{m}{3 k n} \sum_{\alpha=A}^{B} \int\left(c_{\alpha}-v\right)^{2} f_{\alpha} d \boldsymbol{c}_{\alpha}$,

whose time-space evolution is defined by the governing balance equations

$$
\begin{aligned}
\frac{\partial n_{\alpha}}{\partial t}+\sum_{i=1}^{3} \frac{\partial n_{\alpha}}{\partial x_{i}}\left(n_{\alpha} v_{i}+n_{\alpha} u_{i}^{\alpha}\right) & =\tau_{\alpha} \\
\frac{\partial}{\partial t}\left(\varrho v_{i}\right)+\sum_{j=1}^{3} \frac{\partial}{\partial x_{i}}\left(p_{i j}+\varrho v_{i} v_{j}\right) & =0 \\
\frac{\partial}{\partial t}\left(\frac{3}{2} n k T+\sum_{\alpha=A}^{B} n_{\alpha} E_{\alpha}\right. & \left.+\frac{1}{2} \varrho v^{2}\right)+\sum_{i=1}^{3} \frac{\partial}{\partial x_{i}}\left\{q_{i}+p_{i j} v_{j}\right. \\
& \left.+\left(\frac{3}{2} n k T+\sum_{\alpha=A}^{B} n_{\alpha} E_{\alpha}+\frac{1}{2} \varrho v^{2}\right) v_{i}\right\}=0 .
\end{aligned}
$$

Above, $u_{i}^{\alpha}$ is the diffusion velocity of the $\alpha$-constituent, and $\rho, p_{i j}, q_{i}$ are the mass density, pressure tensor and heat flux of the mixture. Moreover, the production term $\tau_{\alpha}$ on the r.h.s. of equation (7) represents the reaction rate of the constituent $\alpha$ and equation (7) represents itself the rate law of the considered reactive system. As it is well known, equations (7-9) become closed when a suitable hydrodynamic closure procedure is applied, in agreement with an appropriate chemical regime, and the constitutive equations for $u_{i}^{\alpha}, p_{i j}, q_{i}$ and $\tau_{\alpha}$ are derived.

Typically, when studying the propagation of steady detonation waves starting from a kinetic frame, an Eulerian regime with fast chemical reaction is assumed. This has been considered in papers [4] and [10], for example, where the assumed chemical regime is defined by the Maxwellian distributions of mechanical equilibrium and the elastic and reactive time scales are of the same order. The only non-equilibrium effect described in this regime is the relaxation of the mixture towards a chemical and thermal equilibrium state. The steady detonation solutions of the above cited papers reproduce the typical behaviour of the wave in the vicinity of the final equilibrium state. Conversely, in the more recent paper [19] by the same authors of the present work, another chemical regime has been considered in order to describe the complete reactive process, starting from its early stage and going towards the equilibrium final state.

In view of the steady detonation problem and linear stability analysis of the next sections, the hydrodynamic closure procedure proposed and explained in paper [6] for the present kinetic modelling is here adopted. The details of the closure procedure, based on the asymptotic method of Chapman-Enskog and a second order Sonine expansion of the distribution functions, are here omitted for sake of brevity. They are given 
in paper [6] and revisited in the book [8]. Accordingly, a chemical regime of slow reactive process is here considered, for which the elastic collisions are more frequent than reactive interactions and elastic time scale is smaller than the reactive one. In this case, the elastic collisions drive the distribution functions towards local quasiequilibrium Maxwellians, whereas the reactive interactions tend to disturb the local quasi-equilibrium distributions. After an appropriate scalling of the kinetic equations (1) consistent with the adopted chemical regime, and solving the integral equations for the expansion corrections, the second-order non-equilibrium distribution functions have been explicitly obtained in paper [6], as a perturbation of the Maxwellian distributions $f_{\alpha}^{M}$, in the following form

$$
\widetilde{f}_{\alpha}=f_{\alpha}^{M}\left[1+\omega\left(\frac{15}{8}-\frac{5 m\left(c_{\alpha}-v\right)^{2}}{4 k T}+\frac{m^{2}\left(c_{\alpha}-v\right)^{4}}{8 k^{2} T^{2}}\right)\right],
$$

with the deviation factor $\omega$ given by

$$
\omega=x_{A}^{2}\left(\frac{\mathrm{d}}{\mathrm{d}_{r}}\right)^{2} \frac{Q_{R}^{\star}}{8}\left(Q_{R}^{\star}+Q_{R}^{\star} \varepsilon_{A}^{\star}-\varepsilon^{\star}+2 \varepsilon_{A}^{\star 2}-1\right) e^{-\varepsilon_{A}^{\star}},
$$

where $x_{A}=n_{A} / n$ is the concentration of the $A$-constituent. The above non-equilibrium distributions $\widetilde{f}_{\alpha}$ of expression (10) exhibit an appreciable influence of the reaction heat $Q_{R}^{\star}$ and forward activation energy $\varepsilon_{A}^{\star}$, so that the departure from the equilibrium state can be evaluated in terms of these chemical kinetic parameters, in particular for both exothermic $\left(Q_{R}^{*}<0\right)$ and endothermic $\left(Q_{R}^{*}>0\right)$ chemical reactions.

The hydrodynamic closure of equations (7-9) is achieved when the non-equilibrium distributions $\tilde{f}_{\alpha}$ given by expressions (10-11) are used to evaluate the reaction rate $\tau_{\alpha}$, constituent diffusion velocities $u_{i}^{\alpha}$, mixture pressure tensor $p_{i j}$ and heat flux $q_{i}$, and the corresponding constitutive equations are derived. The reactive governing equations, resulting from this hydrodynamic closure of equations (7-9), define the macroscopic mathematical analog for the considered reactive mixture with a single reversible chemical reaction. The constitutive laws obtained with the non-equilibrium distributions (10-11) for the constituent diffusion velocities, mixture heat flux and pressure tensor correspond to a non-diffusive, non-heat conducting and non-viscous reactive mixture, namely

$$
u_{i}^{\alpha}=0, \quad q_{i}=0, \quad p_{i j}=p \delta_{i j}, \quad i, j=1,2,3, \quad \alpha=A, B,
$$

where $p$ is the mixture pressure, with $p=n k T$, and $\delta_{i j}$ represents the Kronecker delta. Moreover, the reaction rate $\tau_{\alpha}$ is explicitly given by

$$
\begin{aligned}
\tau_{B}=-\tau_{A}, \quad \tau_{A}= & -4 n_{A}^{2} \mathrm{~d}_{r}^{2} \sqrt{\frac{\pi k T}{m}} e^{-\varepsilon_{A}^{\star}}\left[1+\varepsilon_{A}^{\star}+\frac{x_{A}^{2}}{128}\left(\frac{\mathrm{d}}{\mathrm{d}_{r}}\right)^{2} Q_{R}^{\star}\right. \\
& \left.\times\left(1+Q_{R}^{\star}+Q_{R}^{\star} \varepsilon_{A}^{\star}+\varepsilon_{A}^{\star}-2 \varepsilon_{A}^{\star 2}\right)\left(4 \varepsilon_{A}^{\star 3}-8 \varepsilon_{A}^{\star 2}-\varepsilon_{A}^{\star}-1\right) e^{-\varepsilon_{A}^{\star}}\right] .
\end{aligned}
$$

This form of the reaction rate reproduces an Arrhenius-type chemical kinetics and exhibits the contribution of both the reaction heat $Q_{R}^{*}$ and activation energy $\varepsilon_{A}^{\star}$. It will be crucial for the study developed in sections 3 and 4 about the steady detonation and linear stability. The closed set of the governing balance equations are obtained 
from equations (7-9) and constitutive laws (12-13). In the particular case of one-spacedimensional evolution ( $x$-direction), they can be written in the form

$$
\begin{aligned}
& \frac{\partial n_{\alpha}}{\partial t}+\frac{\partial}{\partial x}\left(n_{\alpha} v\right)=\tau_{\alpha}, \quad \alpha=A, B, \\
& \frac{\partial v}{\partial t}+\frac{1}{\varrho} \frac{\partial p}{\partial x}+v \frac{\partial v}{\partial x}=0, \\
& \frac{\partial p}{\partial t}+v \frac{\partial p}{\partial x}+\frac{5}{3} p \frac{\partial v}{\partial x}+\frac{2}{3} \sum_{\alpha=A}^{B} E_{\alpha} \tau_{\alpha}=0
\end{aligned}
$$

where $v$ represents now the $x$-component of the mixture velocity. The above set of governing equations can be written in a more conservative form, substituting the two evolution equations (14) by the equivalent set

$$
\begin{aligned}
& \frac{\partial n_{A}}{\partial t}+\frac{\partial}{\partial x}\left(n_{A} v\right)=\tau_{A}, \\
& \frac{\partial}{\partial t}\left(n_{A}+n_{B}\right)+\frac{\partial}{\partial x}\left[\left(n_{A}+n_{B}\right) v\right]=0,
\end{aligned}
$$

where equation (18) is a conservation equation for the total number density $n_{A}+n_{B}$. The governing equations are the reactive Euler equations corrected with the effects of the reaction heat, which result to be appropriate for the applications of the next subsections. They have been obtained with the chemical kinetic approach described in this section. In particular, the detailed form of the reaction rate $\tau_{\alpha}$, expressed in equation (13), retains the kinetic origin of the considered modelling.

\section{Steady detonation wave solutions}

In this section, the basic understanding of the detonation phenomena is presented and the main aspects of the steady detonation dynamics are recalled, with the aim of investigating the linear stability of the steady wave solution. A first and preliminary attempt of the present study, regarding the structure of the steady detonation wave, has been presented by the authors in paper [19].

The explosive mixture consists in the binary reactive system of section 2 , involving a reversible chemical reaction of symmetric type, which can be either of exothermic or endothermic type. The study is performed for both cases of ZND and pathologicaltype detonation waves $[21,22]$. The former corresponds to a detonation wave which propagates with velocity greater or equal to the Chapman-Jouguet velocity and is sustained by the energy release of an exothermic chemical reaction. Thus, once initiated, the ZND detonation does not need any external support to sustain its further evolution. The latter pathological-type detonation wave propagates with velocity greater than the Chapman-Jouguet speed and occurs when an endothermic chemical reaction takes place or other dissipative effects are present in the final stage of the chemical process. Typically the system involves two consecutive irreversible chemical reactions with the first one being exothermic and the second one endothermic [29, 30]. However, other complexities in the reactive system, in particular more than one reversible reaction, 
can produce a pathological detonation, as explained in references [22] and [30], for example. In the present analysis, the model reactive system involves one chemical reaction only, which can be either of exothermic or endothermic type. Therefore, the proposed modelling can reproduce the complete configuration of the ZND solution as well as the final endothermic stage of the pathological-type detonation solution.

\subsection{Mathematical formulation of the steady detonation problem}

The reactive governing equations referred to the one-space-dimension and written in the more conservative form (15-18) are used in this section to model a detonating binary mixture undergoing the reversible chemical reaction $A+A \rightleftharpoons B+B$. It is well known that such type of hyperbolic equations admit steady traveling detonation wave solutions which describe a combustion regime in which a strong planar shock wave ignites the mixture and the burning keeps the shock advancing and proceeding to equilibrium behind the shock. The ZND idealized model of the detonation wave gives a good and accepted description of such detonation wave solutions. From the mathematical point of view, the ZND model is based on the reactive Euler equations that are simple in form and capable of describing the relevant chemical kinetics of the detonation wave. The configuration of the ZND wave consists of a leading, planar, non-reactive shock wave propagating with constant velocity, followed by a finite reaction zone where the chemical reaction takes place. See figure 1 . The shock wave is assumed to propagate in

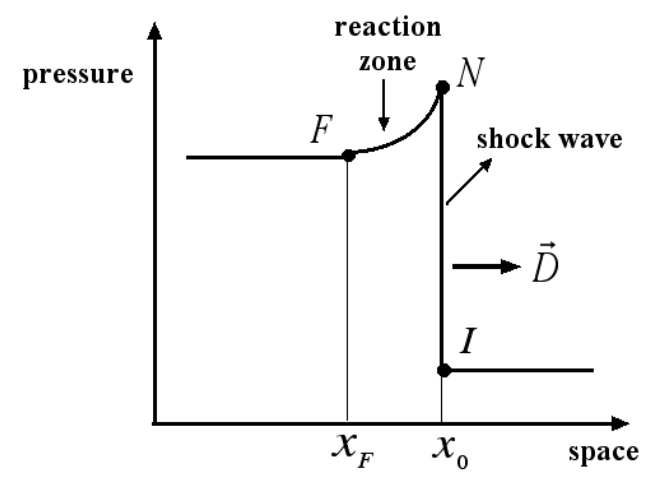

Figure 1. ZND configuration of a steady detonation wave profile for the mixture pressure.

the $x$-direction, from left to right, with velocity $D$. In the steady frame attached to the shock wave, the reaction zone remains from $x_{0}$ to $x_{F}$, where $x_{0}$ represents the location of the shock front. The state just behind the shock, located at $x=x_{0}$, is the Von Neumann state $N$, where the chemical reaction is triggered, and the one located at $x=x_{F}$, at the end of the reaction zone, is the final state $F$, where the chemical reaction reaches the equilibrium. Ahead of the shock front, that is for $x>x_{0}$, the quiescent mixture is at rest in its initial state $I$, where the rate of the chemical reaction is negligible. Inside the reaction zone, for $x_{F}<x<x_{0}$, the mixture evolves through their intermediate states $R$ of partial reaction until reaching the final state. Since the entire wave configuration 
is steady in the shock attached frame, a new reference frame moving with the shock is considered and the normalized steady variable $x_{s}$ is introduced (see figure 1)

$$
x_{s}=\frac{x-D t}{D t_{c}}, \quad t_{c}=\frac{1}{4 n^{+} \mathrm{d}^{2}} \sqrt{\frac{m}{\pi k T^{+}}},
$$

where the superscript + refers to the initial state $I$ and $t_{c}$ is a characteristic time. For sake of simplicity, the normalized steady variable $x_{s}$ will be still denoted with the plane symbol $x$. Governing equations (15-18) are then transformed to the steady frame attached to the shock wave through the transformation (19). Writing $x$ in place of $x_{s}$, they assume the form

$$
\begin{aligned}
& \frac{d}{d x}\left[(v-D) n_{A}\right]=D t_{c} \tau_{A}, \\
& \frac{d}{d x}\left[(v-D)\left(n_{A}+n_{B}\right)\right]=0, \\
& \frac{d}{d x}[(v-D) \varrho v+n k T]=0, \\
& \frac{d}{d x}\left[(v-D)\left(\frac{3}{2} n k T+\frac{\varrho v^{2}}{2}+E_{A} n_{A}+E_{B} n_{B}\right)+n k T v\right]=0,
\end{aligned}
$$

where $\tau_{A}$ is given by expression (13).

\subsection{One-dimensional steady states}

The spatial structure of the ZND detonation wave is determined by means of the Rankine-Hugoniot conditions, connecting the fluxes of the macroscopic quantities preserved across the shock front, together with the rate law, describing the advancement of the chemical process in the reaction zone. Accordingly, the conservative ODEs (21-23) are integrated across the shock front, between the quiescient initial state $\left(n_{A}^{+}, n_{B}^{+}, 0, T^{+}\right)$ and an arbitrary state $\left(n_{A}(x), n_{B}(x), v(x), T(x)\right), x \in\left[x_{F}, x_{0}\right]$, within the reaction zone, leading to the Rankine-Hugoniot conditions, namely

$$
\begin{aligned}
n_{B}\left(n_{A}\right)= & \frac{\left(n_{B}^{+}+n_{A}^{+}\right) D}{D-v}-n_{A} \\
T\left(n_{A}\right)= & \frac{(D-v)\left(\varrho^{+} D v+n^{+} k T^{+}\right)}{n^{+} k D} \\
v\left(n_{A}\right)= & \frac{2 Q_{R}^{*} n_{A}+3 \varrho^{+} D^{2}-5 n^{+} k T^{+}}{8 \varrho^{+} D} \\
& +\frac{\sqrt{\left(2 Q_{R}^{*} n_{A}+3 \varrho^{+} D^{2}-5 n^{+} k T^{+}\right)^{2}-32 \varrho^{+} Q_{R}^{*} D^{2}\left(n_{A}-n_{A}^{+}\right)}}{8 \varrho^{+} D}
\end{aligned}
$$

The rate law results from equation (20) in the form

$$
\frac{d n_{A}}{d x}=\frac{D t_{c} \tau_{A}}{v-D+n_{A} \frac{d v}{d n_{A}}},
$$

and gives the $x$-evolution of the constituent number density $n_{A}$ in the reaction zone, specifying the chemical composition of the explosive mixture. Equations (24-27), with 
$D$ and $Q_{R}^{*}$ as parameters, characterize any arbitrary state within the reaction zone in dependence of the initial state. When a negative value of $Q_{R}^{*}$ is considered, the chemical reaction is exothermic and the structure of the resulting wave solution reproduces the ZND configuration. Conversely, when a positive value of $Q_{R}^{*}$ is considered, the chemical reaction is endothermic and the structure of the resulting wave solution replicates the configuration of the last stage of the pathological-type detonation. The methodology for solving the above equations (24-27) comprises the following two steps.

Step 1. The von Neumann state $N$, just ahead the shock, is characterized by equations (24-26) together with a further jump condition of Rankine-Hugoniot type, which is consistent with the still unreacted character of the von Neumann state. This RK-condition is obtained by integrating across the shock the further conservative ODE resulting from the rate equation (27) with $\tau_{A}$ settled equal to zero.

Step 2. All intermediate states inside the reaction zone $\left(x_{F}<x<x_{0}\right)$, as well as the final state at the end of the reaction zone $\left(x=x_{F}\right)$, are obtained by integrating the rate equation (27) with initial condition at the von Neumann state, and then solving the algebraic equations (24-26) for the considered state. In particular, the equilibrium final state is obtained when the above referred integration gives a vanishing value for the reaction rate $\tau_{A}$.

This problem is numerically solved for both types of exothermic and endothermic chemical reaction, and some simulations are performed for one elementary reaction of the chain branching of a theoretical detonating mixture. The detonation velocity $D$ and the kinetic and thermodynamical reference input parameters are assumed as follows

$$
\begin{aligned}
& D=1700 \mathrm{~ms}^{-1}, \quad n_{A}^{+}=0.35 \mathrm{~mol} / \mathrm{l}, \quad n_{B}^{+}=0 \mathrm{~mol} / \mathrm{l}, \\
& m=0.01 \mathrm{Kg} / \mathrm{mol}, \quad T^{+}=298.15 \mathrm{~K}, \quad E_{A}=2400 \mathrm{~K}, \quad \varepsilon_{A}^{\star}=6 .
\end{aligned}
$$
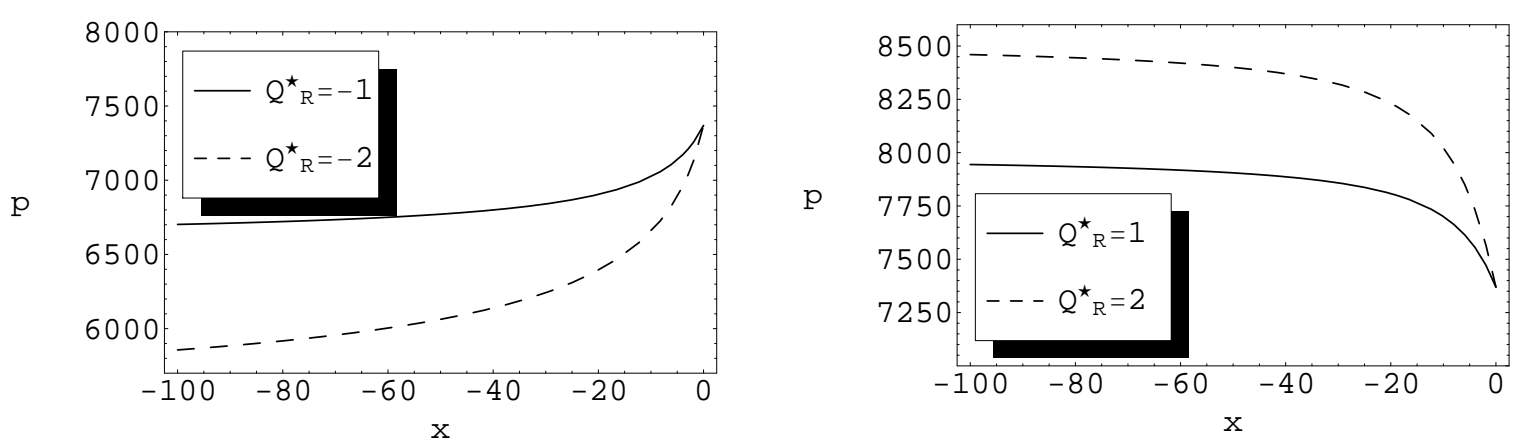

Figure 2. Steady detonation profile for the mixture pressure $p$. Left: exothermic chemical reaction with $Q_{R}^{\star}=-1$ (solid line) and $Q_{R}^{\star}=-2$ (dashed line). Right: endothermic chemical reaction with $Q_{R}^{\star}=1$ (solid line) and $Q_{R}^{\star}=2$ (dashed line). 

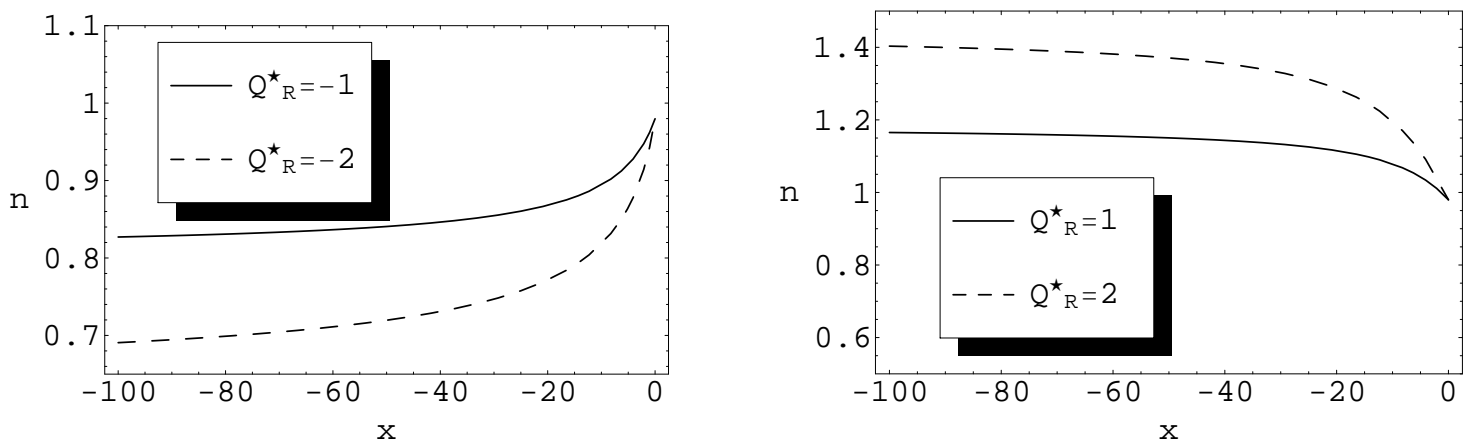

Figure 3. Steady detonation profile for the mixture number density $n$. Left: exothermic chemical reaction with $Q_{R}^{\star}=-1$ (solid line) and $Q_{R}^{\star}=-2$ (dashed line). Right: endothermic chemical reaction with $Q_{R}^{\star}=1$ (solid line) and $Q_{R}^{\star}=2$ (dashed line).
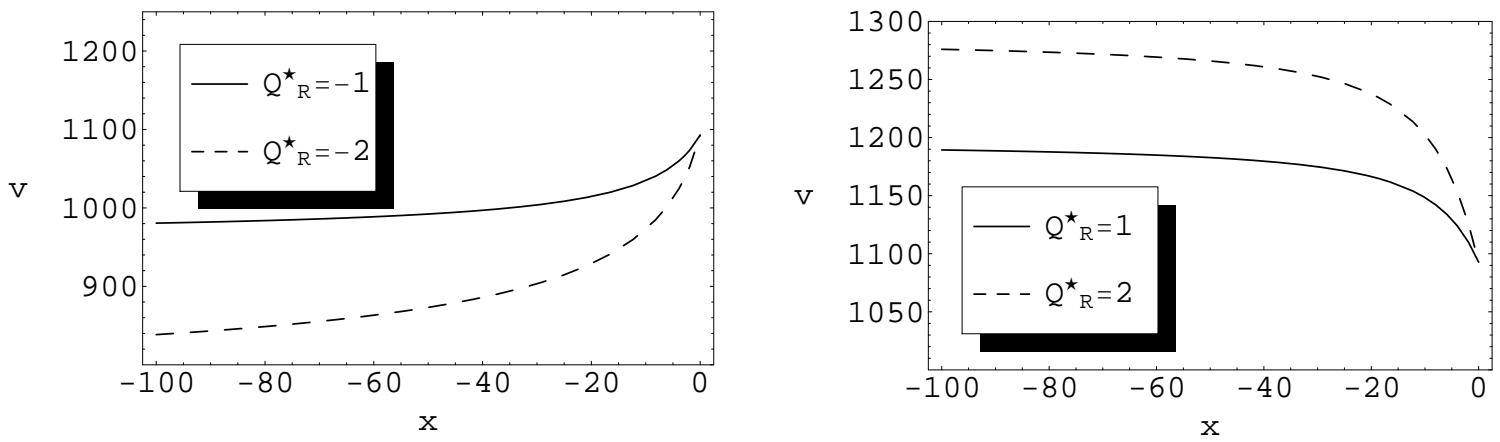

Figure 4. Steady detonation profile for the mixture mean velocity $v$. Left: exothermic chemical reaction with $Q_{R}^{\star}=-1$ (solid line) and $Q_{R}^{\star}=-2$ (dashed line). Right: endothermic chemical reaction with $Q_{R}^{\star}=1$ (solid line) and $Q_{R}^{\star}=2$ (dashed line).
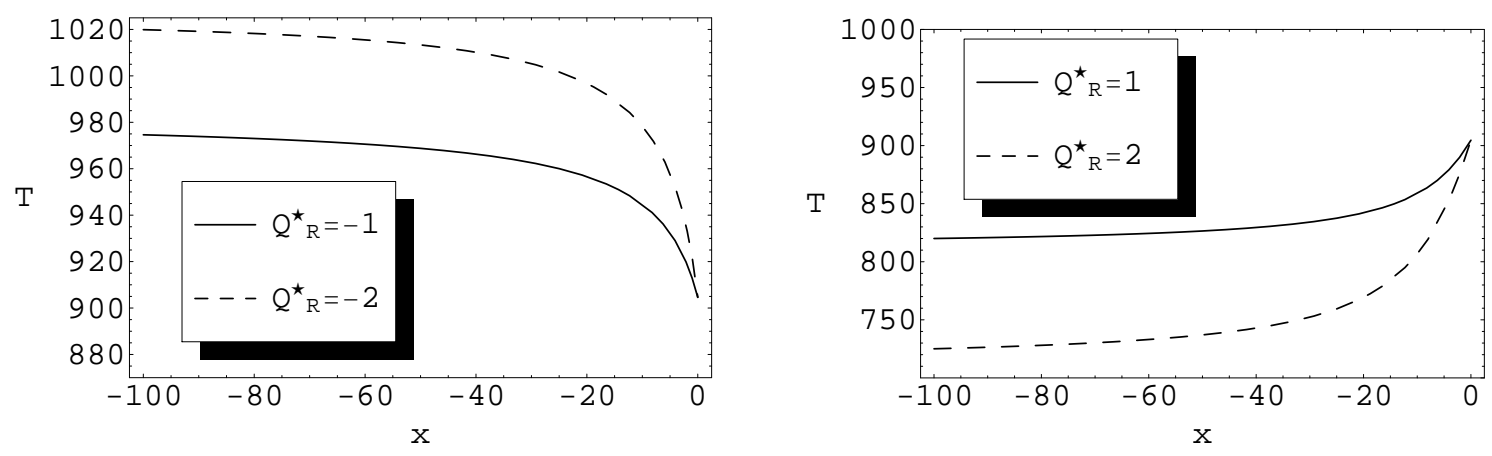

Figure 5. Steady detonation profile for the mixture temperature T. Left: exothermic chemical reaction with $Q_{R}^{\star}=-1$ (solid line) and $Q_{R}^{\star}=-2$ (dashed line). Right: endothermic chemical reaction with $Q_{R}^{\star}=1$ (solid line) and $Q_{R}^{\star}=2$ (dashed line).

Figures 2, 3, 4 and 5 show some representative steady detonation profiles for the mixture pressure $p$, number density $n$, mean velocity $v$ and temperature $T$, respectively, in dependence of the algebraic distance behind the shock wave. The left frames of figures 2, 3, 4 and 5 refer to two exothermic chemical reactions with reaction heat $Q_{R}^{*}=-2$ and 
$Q_{R}^{*}=-1$. The right frames refer to two endothermic chemical reactions with reaction heat $Q_{R}^{*}=1$ and $Q_{R}^{*}=2$. The $p, n$ and $v$ profiles of figures 2,3 and 4 show that the steady detonation solution is a reactive rarefaction wave for an exothermic reaction (left frames) and a reactive compression wave for an endothermic reaction (right frames). On the other hand, one can observe in figure 5 that the temperature increases for an exothermic chemical reaction (left frame) and decreases for an endothermic reaction (right frame), as expected. The left frames reproduce the typical configuration of an ZND steady detonation wave arising in a real explosive system with one exothermic chemical reaction $[21,22]$. On the other hand, the right frames replicate the essential features of the dynamics of the endothermic stage of a typical chain-branching reactive system with pathological detonation, more specifically for what concerns the branch between the so called pathological point and the strong final state [21, 22, 29, 30]. The representation of the wave structure is supplemented with the variation of the reaction zone thickness with the reaction heat $Q_{R}^{*}$, reported on table 1 . One can extract from this table that the extent of the reaction zone decreases when the reaction heat $Q_{R}^{*}$ increases. This is an expected feature, in agreement with other numerical and experimental works, see paper [41], for example, for what concerns exothermic reactions.

Table 1. Variation of the reaction zone thickness with the reaction heat $Q_{R}^{*}$, in the range $-2 \leq Q_{R}^{*} \leq 2$. Left: exothermic chemical reaction (ZND detonation). Right: endothermic chemical reaction (final stage of the pathological-type detonation).

\begin{tabular}{ll}
\hline$Q_{R}^{*}$ & reaction zone thickness \\
\hline-2 & 4584.52 \\
-1.75 & 4459.53 \\
-1.5 & 4354.21 \\
-1.25 & 4261.35 \\
-1 & 4177.18 \\
-0.5 & 4026.60 \\
\hline
\end{tabular}

\begin{tabular}{ll}
\hline$Q_{R}^{*}$ & reaction zone thickness \\
\hline 0 & 3891.85 \\
0.25 & 3828.57 \\
0.5 & 3767.36 \\
1 & 3649.74 \\
1.5 & 3536.66 \\
2 & 3426.36 \\
\hline
\end{tabular}

\section{One-dimensional linear stability analysis}

It is well known from theoretical studies as well as from experimental investigations and numerical simulations, that the ZND detonation wave with an extended reaction zone can be dynamically unstable to small perturbations and exhibit a significant unsteady structure, especially in gaseous mixtures [13, 21, 22, 29, 32, 37, 38, 43, 48, 49, 50]. This means that the steady solution can degenerate into an oscillatory solution in the longtime limit. Typically, it results to be very difficult to determine, analytically or even numerically, such oscillatory solution, because it exhibits complex three-dimensional non-linear perturbations.

The standard preliminary step of a formal treatment is a hydrodynamical stability analysis of the steady detonation solution. This consists in assuming that a small 
rear boundary perturbation is instantaneously assigned and induces a deviation on the shock wave position, giving rise to small perturbations on the state variables, which propagate in the reaction zone. The steady configuration of the detonation wave solution is then affected and the evolution of the state variables perturbations in the reaction zone determines the stability of the steady detonation solution. In fact, when any perturbation grows with time, the steady solution becomes hydrodynamically unstable, and if all perturbations decay in time, the steady solution becomes stable. The stability analysis provides important information about the instability modes, growth rate perturbations, neutral stability boundaries and other features of the stability behaviour in the parameter space.

From the mathematical point of view, the hydrodynamical stability problem requires the transformation to the perturbed shock attached frame, and then the linearization of the governing equations and Rankine-Hugoniot shock conditions around the steady detonation solution. This will be done in the next subsection, adopting the normal mode approach proposed by Lee and Stewart in paper [37] and then followed by several authors, see for example paper [18] and the references therein cited.

\subsection{Formulation of the linear stability problem}

The hydrodynamical stability of the steady detonation solution characterized in terms of the Rankine-Hugoniot conditions (24-26) and rate law (27), as described in section 3 , is investigated here adopting a normal mode approach to determine one-dimensional instability modes. The linear stability problem is formulated as an initial-boundary value problem in terms of the stability differential equations, with initial conditions at the von Neumann state and an additional closure boundary condition at the final state.

Stability equations. The one-dimensional closed governing equations (14-16) are transformed first to the perturbed wave coordinate $x$, which measures the distance from the perturbed shock, namely

$$
x=x^{\ell}-\psi(t), \quad \text { with } \quad \psi(t)=D t+\widetilde{\psi}(t),
$$

where $x^{\ell}$ is the laboratory frame coordinate, $D$ the constant velocity of the unperturbed shock, $\widetilde{\psi}(t)$ the displacement of the shock wave from the unperturbed position due to a small perurbation, and $\psi(t)$ the location of the perturbed wave. In the new shock attached coordinate system, the instantaneous position of the perturbed shock wave is $x=0$ and its velocity is $D(t)=D+\widetilde{\psi}^{\prime}(t)$. Further, a normal mode expansion with exponential time dependent perturbations is assumed for the steady state variables,

$$
z(x, t)=z^{*}(x)+e^{a t} \bar{z}(x), \quad \psi(t)=\bar{\psi} e^{a t}, \quad a, \bar{\psi} \in \mathbb{C},
$$

where $z=\left[\begin{array}{llll}n_{A} & n_{B} & v & p\end{array}\right]^{T}$ is the state vector, $z^{*}(x)$ indicates the one-dimensional steady solution, $\bar{z}(x)$ is the vector of complex eigenfunctions representing the unknown spatially disturbances, $\bar{\psi}$ is a complex disturbance amplitude parameter and $a$ is the complex eigenvalue, with Re $a$ and $\operatorname{Im} a$ being the disturbance growth rate and frequency, 
respectively. Since the assigned perturbations are small, the transformed governing equations in the perturbed shock frame are linearized about the steady solution $z^{*}(x)$, by means of the expansions (30). Performing a further normalization of the state variables with respect to the complex amplitude parameter $\bar{\psi}$, namely $\bar{w}=\bar{z} / \bar{\psi}$, one obtains the evolution equations in the wave coordinate $x$, for the complex disturbances. Rewriting $\bar{z}$ instead of $\bar{w}$, the resulting equations, for $x \in] x_{F}, 0[$, are

$$
\begin{aligned}
& D a \bar{n}_{\alpha}+\left(v^{*}-D\right) \frac{d \bar{n}_{\alpha}}{d x}+\frac{n_{\alpha}^{*}}{d x}(\bar{v}-D a)+\frac{d v^{*}}{d x} \bar{n}_{\alpha}+n_{\alpha}^{*} \frac{d \bar{v}}{d x}=\bar{\tau}_{\alpha}, \quad \alpha=A, B, \\
& \varrho^{*} D a \bar{v}+\frac{d \bar{p}}{d x}+\varrho^{*} \frac{d v^{*}}{d x}(\bar{v}-D a)+\left(v^{*}-D\right) \frac{d v^{*}}{d x} \bar{\varrho}+\varrho^{*}\left(v^{*}-D\right) \frac{d \bar{v}}{d x}=0, \\
& D a \bar{p}+\frac{5}{3}\left(p^{*} \frac{d \bar{v}}{d x}+\bar{p} \frac{d v^{*}}{d x}\right)+\left(v^{*}-D\right) \frac{d \bar{p}}{d x}+(\bar{v}-D a) \frac{d p^{*}}{d x}=\frac{Q_{R}^{*} D t_{c} \bar{\tau}_{A}}{3},
\end{aligned}
$$

with $\bar{\tau}_{\alpha}$ being the linearized reaction rate, given by

$$
\begin{aligned}
\bar{\tau}_{B}=-\bar{\tau}_{A}, & \\
\bar{\tau}_{A}=-4 d_{r}^{2} \sqrt{\frac{\pi k}{m}} e^{-\epsilon^{\star}} & {\left[\left(2 n_{A}^{*} \bar{n}_{A} \sqrt{T^{*}}+\frac{\bar{p}+\frac{\bar{n}}{n^{*}} p^{*}}{2 n^{*} k \sqrt{T^{*}}} n_{A}^{* 2}\right)\left(1+\epsilon^{\star}+\Gamma x_{A}^{* 2}\right)\right.} \\
& \left.+2 \sqrt{T^{*}} \frac{n_{A}^{*}{ }^{3}}{n^{*}}\left(-n_{A}^{*} \bar{n}_{B}+n_{B}^{*} \bar{n}_{A}\right)\right],
\end{aligned}
$$

where $T^{*}=k n^{*} p^{*}$ is the steady mixture temperature and $\Gamma$ is given by

$$
\Gamma=\frac{1}{128}\left(\frac{d}{d_{r}}\right)^{2} Q_{R}^{\star}\left(1+Q_{R}^{\star}+Q_{R}^{\star} \varepsilon_{A}^{\star}+\varepsilon_{A}^{\star}-2 \varepsilon_{A}^{\star 2}\right)\left(4 \varepsilon_{A}^{\star 3}-8 \varepsilon_{A}^{\star 2}-\varepsilon_{A}^{\star}-1\right) e^{-\varepsilon_{A}^{\star}} .
$$

Equations (31-33) constitute the stability equations for the present modelling, giving the spatial evolution of the complex perturbations $\bar{z}(x)$ in the reaction zone, from the perturbed shock position $x=0$ to the equilibrium final state $x=x_{F}$. They constitute a system of eight first-order homogeneous linear ordinary differential equations with spatially varying coefficients, for the real and imaginary parts of the complex perturbations.

Initial conditions. The initial conditions to be joined to the stability equations (3133) are the linearized perturbed Rankine-Hugoniot relations which connect the value of the disturbances at the von Neuman state to their zero value ahead the perturbed shock. They are provided by the Rankine-Hugoniot relations (24-26) together with the further jump condition for the von Neuman state obtained as explained in Subsection 3.1. After transforming to the wave coordinate, linearizing around the steady state and normalizing with respect to $\bar{\psi}$, the resulting jump conditions at the von Neuman state are obtained in the form

$$
\begin{aligned}
& \bar{n}_{\alpha}(0)=\frac{\left(n_{\alpha}^{*}-n_{\alpha}^{+}\right) a-n_{\alpha}^{*} \bar{v}(0)}{v^{*}-D}, \quad \alpha=A, B, \\
& \bar{v}(0)=\frac{3 \varrho^{+} v^{* 2}+\frac{3}{2}\left(p^{*}-p^{+}\right)-\frac{3}{2} D \varrho^{+} v^{*}+2 E_{A} n^{+}+Q_{R}^{*} n_{B}^{+}}{-\varrho^{*}\left(v^{*}-D\right)^{2}+\frac{5}{2} p^{*}} a \\
& \bar{p}(0)=-\varrho^{+} a v^{*}-\left(v^{*}-D\right) \varrho^{*} \bar{v}(0) .
\end{aligned}
$$


Equations (36-38) give the initial conditions for the stability equations (31-33). However, the stability system is not closed, since the complex growth rate $a$ is involved.

Closure condition. The required closure condition, which gives the dispersion relation for the normal modes (30), is the acoustics radiation condition adopted in many previous works on detonation stability as, for example, in papers [10, 18, 34, 37, 41, 42, 47]. Such condition states that the inherent instability of the detonation wave solution results exclusively from the interplay between the leading shock and the reaction zone and can not be affected by further disturbances traveling towards the shock from a great distance from the reaction zone. Thus the closure condition is a boundary condition assigned at the equilibrium final state as

$$
\bar{v}\left(x_{F}\right)+a=\frac{-1}{\gamma \varrho_{e q}^{*} c_{e q}^{*}} \bar{p}\left(x_{F}\right),
$$

where $\gamma$ is the ratio of specific heats, $c_{e q}^{*}$ and $\varrho_{e q}^{*}$ the isentropic sound speed and gas density at the equilibrium final state, for $x=x_{F}$. Equation (39) is usually regarded as the necessary condition to define the dynamics of the complex growth rate $a$. It was originally derived in paper [34] through an acoustic analysis performed at the end of the reaction zone. Important discussions about the closure condition and detailed derivations can be found in papers [18, 37, 42], for example. These papers show that the closure condition can be alternatively derived following two distinct approaches, one being physically based on an accurate acoustic analysis and another one being justified by a boundedness condition which requires that the asymptotic structure of the perturbed solution is independent of elementary unbounded solutions. Different closure conditions can be imposed to assure the determinacy of the stability problem, as for example a piston-type condition which requires the vanishing of the velocity perturbation at a piston located far downstream of the shock wave. However, as discussed in paper [42], the further interaction of the piston with the shock wave would alter the instability spectrum leading to different stability results in comparison to those coming from the intrinsic mechanism between the shock wave and the reaction zone.

Concluding this subsection, the one-dimensional linear stability problem of the steady detonation is formulated in terms of the complex disturbances $\bar{z}(x)$ and complex growth rate $a$, by means of the ordinary differential equations (31-33) for $x \in] x_{F}, 0[$, with initial conditions (36-38) at $x=0$ and closure condition (39) at $x=x_{F}$. This problem is treated numerically as described in the next subsections.

\subsection{Discussion on the numerical scheme}

The stability problem is treated numerically with the aim of obtaining an extensive investigation of the stability spectrum for the eigenfunction perturbations $\bar{z}$ and eigenvalue perturbation parameter $a$, in terms of the parameters characterizing the steady solution. For a given set of thermodynamical and chemical parameters describing the steady detonation solution, the disturbances $\bar{z}(x)$ and perturbation parameter $a$ are 
determined applying an iterative shooting technique based on the numerical method proposed by Lee and Stewart in paper [37]. Accordingly, a trial value of $a$ in a fixed bounded domain $\mathcal{R}$ of the complex plane is considered and then equations (31-33) are integrated in the reaction zone $] x_{F}, 0$ [ with initial conditions (36-38) at $x=0$, using a fourth order Runge-Kutta routine. The solution $\bar{z}(x), x \in\left[x_{F}, 0\right]$, obtained for the considered trial value of $a$ is then specialized for $x=x_{F}$ to inquire if the boundary condition (39) is verified. However, for a given steady detonation solution, an arbitrary value of $a$ does not satisfy the closure condition (39) and thus it does not produce a solution of the stability problem. To overcome this difficulty, the residual function $\mathscr{H}(a)$, defined from the closure condition (39) by the expression

$$
\mathscr{H}(a)=\bar{v}\left(x_{F}\right)+a+\frac{1}{\gamma \varrho_{e q}^{*} c_{e q}^{*}} \bar{p}\left(x_{F}\right), \quad a \in \mathcal{R},
$$

is estimated at each trial value of $a$, and only those solutions $\bar{z}(x)$ obtained for values of $a$ for which the residual function $\mathscr{H}(a)$ vanishes within a given tolerance are accepted. The search for trial values of the complex parameter a constitutes the key problem in the stability analysis. There exist some numerical techniques to search for these values, as for example those used by Erpenbeck in papers [32, 33] and by Lee and Stewart in paper [37]. In the present study, a different numerical scheme is proposed, recovering the Erpenbeck's idea of counting the number of zeros of $\mathscr{H}$ in a fixed domain of the complex plane, combined with the sooting method proposed by Lee and Stewart. First, a confidence domain $\mathcal{R}$ in the complex plane, containing at least one zero of $\mathscr{H}$, is found and successively refined in order to reduce the computational effort in the next calculations. Then, a three-dimensional plot of $|\mathscr{H}|$ is constructed in the considered refinement of $\mathcal{R}$ and the zeros of $\mathscr{H}$ are finally identified. In Subsection 4.3, the numerical scheme is explained in detail.

\subsection{Numerical solution and technique}

Instability modes correspond to a positive growth rate Re $a$, so that the zeros of the residual function $\mathscr{H}$ are serached in a domain $\mathcal{R}$ on the right half of the complex plane. On the other hand, since these modes occur in conjugate pairs, it is enough to choose a domain $\mathcal{R}$ in the upper-right quarter of the complex plane.

The numerical method proposed in this paper provides a rapid and efficient procedure to investigate if the domain $\mathcal{R}$ contains any zero of $\mathscr{H}$, meaning that the corresponding detonation solution is unstable. Moreover, the effective determination of the approximate locations of the zeros of $\mathscr{H}$ in the domain $\mathcal{R}$, and the identification of the corresponding growth rate $\operatorname{Re} a$ and frequency $\operatorname{Im} a$, requires a further refinement of the domain $\mathcal{R}$ as well as a three-dimensional plot of $|\mathscr{H}|$ in the refinement.

Preliminaries. The argument principle used by Erpenbeck in paper [33], combined with the shooting method proposed by Lee and Stewart in paper [37], is adopted here to count the number of zeros of the residual function $\mathscr{H}$ and approximate their location. 
This principle states that the difference between the number $Z$ of zeros and $P$ of poles of the function $\mathscr{H}$ within the region $\mathcal{R}$, provided that there are no zeros in its contour, is given by

$$
Z-P=\frac{1}{2 \pi i} \int_{\zeta} \frac{\mathscr{H}^{\prime}(u)}{\mathscr{H}(u)} d u
$$

or equivalently by

$$
Z-P=\frac{1}{2 \pi i} \int_{k}^{\ell} \frac{\mathscr{H}^{\prime}(\zeta(t))}{\mathscr{H}(\zeta(t))}\left\|\zeta^{\prime}(t)\right\| d t,
$$

where $\zeta:[k, \ell] \rightarrow \mathbb{C}$ is a path smooth by parts, describing the contour of $\mathcal{R}$ in the positive sense. Since $\mathscr{H}$ has no poles in the complex plane, one has $P=0$ and the expression (42) gives the number of zeros of $\mathscr{H}$ inside the region $\mathcal{R}$,

$$
Z=\frac{1}{2 \pi i} \int_{k}^{\ell} \frac{\mathscr{H}^{\prime}(\zeta(t))}{\mathscr{H}(\zeta(t))}\left\|\zeta^{\prime}(t)\right\| d t .
$$

Then, after counting the zeros of $\mathscr{H}$ inside the region $\mathcal{R}$ as well as inside successive refinements of $\mathcal{R}$, a three-dimensional plot of $|\mathscr{H}|$ in the last refinement allows to locate the zeros of $\mathscr{H}$.

It is important to note that the requirement that the residual function $\mathscr{H}$ has no zeros in the contour of $\mathcal{R}$ does not constitute an actual limitation for the application of expressions (43) in the present numerical computation. In fact, the method starts with the residual values $\mathscr{H}\left(a_{j}\right)$ for a very huge number of points $a_{j}$ in the contour of $\mathcal{R}$. If there is any zero of $\mathscr{H}$ in the contour of $\mathcal{R}$ then, at least one of the considered points $a_{j}$ should be close enough to this zero and the location of such point allows to identify the zero without the need of using any further strategy.

General description of the numerical technique. The starting point for the implementation of the numerical technique is the random selection of a great number of trial values for the perturbation parameter $a$ in the contour of a fixed domain $\mathcal{R}$, say $a_{j}, j=1,2, \ldots, n$, such that $a_{j}=\zeta\left(t_{j}\right)$, for $j=1,2, \ldots, n$. Then the integral in expression (43) is estimated using a rather cumbersome procedure. More in detail, the mean value theorem gives

$$
\int_{k}^{\ell} \frac{\mathscr{H}^{\prime}(\zeta(t))}{\mathscr{H}(\zeta(t))}\left\|\zeta^{\prime}(t)\right\| d t=\mu(\ell-k),
$$

where $\mu$ represents the mean value in the interval $[k, \ell]$ of the function $h:[k, \ell] \rightarrow \mathbb{R}$ defined by

$$
h(t)=\frac{\mathscr{H}^{\prime}(\zeta(t))}{\mathscr{H}(\zeta(t))}\left\|\zeta^{\prime}(t)\right\|, \quad t \in[k, \ell]
$$

The mean value $\mu$ of $h$, in turn, is approximated with the mean value $\mu_{S}$ of the set

$$
S=\left\{\frac{\mathscr{H}^{\prime}\left(\zeta\left(t_{j}\right)\right)}{\mathscr{H}\left(\zeta\left(t_{j}\right)\right)}\left\|\zeta^{\prime}\left(t_{j}\right)\right\|: j=1,2, \ldots, n\right\} .
$$


The derivative $\mathscr{H}^{\prime}\left(\zeta\left(t_{j}\right)\right)=\mathscr{H}^{\prime}\left(a_{j}\right)$ is estimated by choosing a suitable point close enough to $a_{j}$, say $b_{j}$, with $\operatorname{Re} b_{j}=\operatorname{Re} a_{j}+10^{-6}$ and $\operatorname{Im} a_{j}=\operatorname{Im} b_{j}$, as follows

$$
\mathscr{H}^{\prime}\left(a_{j}\right) \approx \frac{\mathscr{H}\left(b_{j}\right)-\mathscr{H}\left(a_{j}\right)}{b_{j}-a_{j}}, \quad j=1,2, \ldots, n .
$$

Moreover, it is well known that if $n$ is large enough, then the mean value of the sample $S, \mu_{S}$, can be treated as a statistical variable following a normal distribution with mean value $\mu$ and standard deviation $\sigma_{S} / \sqrt{n}$, with $\sigma_{S}$ being the standard deviation of $S$. Therefore, the mean value $\mu$ of the function $h$ can be inferred in a confidence interval by the mean value $\mu_{S}$ of the sample $S$. The amplitude of the confidence interval can be reduced enlarging, as much as necessary, the number $n$ of points in the set $S$. In the present paper, all the calculations have been performed with a confidence level of $99 \%$. Consequently, the number of zeros of the residual function $\mathscr{H}$ inside the domain $\mathcal{R}$ is estimated as follows

$$
\frac{\ell-k}{2 \pi i}\left(\mu_{S}-2.58 \frac{\sigma_{S}}{\sqrt{n}}\right)<Z<\frac{\ell-k}{2 \pi i}\left(\mu_{S}+2.58 \frac{\sigma_{S}}{\sqrt{n}}\right) .
$$

Finally, the location of the zeros of $\mathscr{H}$ inside the domain $\mathcal{R}$ is determined resorting to a three-dimensional plot of $|\mathscr{H}|$ in a suitable refinement of $\mathcal{R}$. Even if the domain $\mathcal{R}$ is small, the three-dimensional plot of $|\mathscr{H}|$ requires a large computational effort.

Details on the numerical technique. Having the above preliminary ideas in mind, the numerical technique used to solve the stability problem comprises the following steps, as described bellow.

Step 1 (choice of the domain $\mathcal{R}$ ). A bounded domain $\mathcal{R}$ in the upper-right complex plane is considered, and a path $\zeta:[k, \ell] \rightarrow \mathbb{C}$, which is smooth by parts and describes the contour of $\mathcal{R}$ in the positive sense, is fixed.

Step 2 (selection of the trial values for a). A great number of points, say $a_{j}$ with $j=1, \ldots, n$, are selected at random in the contour of $\mathcal{R}$. For each point $a_{j}$, one determines the unique point $t_{j} \in[k, \ell]$ such that $a_{j}=\zeta\left(t_{j}\right)$. Moreover, for each point $a_{j}$ one chooses another point close enough, say $b_{j}$, such that $\operatorname{Re} b_{j}=\operatorname{Re} a_{j}+10^{-6}$ and $\operatorname{Im} a_{j}=\operatorname{Im} b_{j}$, for $j=1, \ldots, n$.

Step 3 (integration of the ODE's). Assuming each point $a_{j}$ and $b_{j}$, for $j=1, \ldots, n$, as a trial value for the perturbation parameter $a$, the differential equations (31-33) are integrated with initial conditions (36-38), using a fourth order Runge-Kutta routine.

Step 4 (evaluation of the residual function). The solutions $\bar{z}(x), x \in\left[x_{F}, 0\right]$, obtained in the previous step for the considered trial values $a_{j}$ and $b_{j}$ are used to evaluate the residual function $\mathscr{H}$ defined by expression (40) at each point $a_{j}$ and $b_{j}$, for $j=1, \ldots, n$.

Step 5 (estimation of the derivative of the residual function). The derivative $\mathscr{H}^{\prime}\left(a_{j}\right)$ is estimated with the quotient between the differences $\mathscr{H}\left(b_{j}\right)-\mathscr{H}\left(a_{j}\right)$ and $b_{j}-a_{j}$, as indicated in equation (47). 
Step 6 (mean value of the sample $S$ ). The mean value $\mu_{S}$ of the sample $S$ introduced in expression (46) is evaluated as the mean value of a statistical variable following a normal distribution with standard deviation given by $\sigma_{S} / \sqrt{n}$, where $\sigma_{S}$ is the standard deviation of $S$

Step 7 (mean value of the function $h$ ). The mean value $\mu$ of the function $h$ defined by expression (45) is inferred from the mean value $\mu_{S}$ of the sample $S$, using a $99 \%$ confidence interval.

Step 8 (estimation of the number of zeros of $\mathscr{H}$ ). The integral in equation (43) is approximeted by the quantity $(k-\ell) \mu$. The number of zeros of the residual function $\mathscr{H}$ within the region $\mathcal{R}$ is approximated using the estimation (48). The amplitude of the interval can be controlled by the number $n$ of points in the set $S$ in such a way that there is only one integer in the interval.

Step 9 (location of the zeros of $\mathscr{H}$ ). A three-dimensional plot of the magnitude of the residual function $\mathscr{H}$ is drawn in a suitable refinement of the domain $\mathcal{R}$, in order to approximate the location of the zeros of $\mathscr{H}$.

For a given set of thermodynamical and chemical parameters, and considering certain bounds for the perturbation parameter $a$, the numerical method described above has been applied to investigate the linear stability of the steady detonation solution. The main objective is to describe the structure of unstable detonation waves and provide more detailed information about the instability parameter regimes. Some numerical simulations have been performed and several visualizations are provided in the next section.

\section{Results and discussion}

The response of the steady detonation solution to the rear boundary perturbations, as well as the influence of the reaction heat on the stability spectra, is investigated numerically. The stability problem formulated in terms of perturbation parameter $a$ and spatially disturbances $\bar{z}$ by the ODE's (31-33) with initial conditions (36-38) and closure boundary condition (39) is treated with the numerical shooting technique described in subsection 4.3. A rectangular domain $\mathcal{R}$ in the upper-right complex plane is considered in order to locate the unstable modes, namely $0.001<\operatorname{Re}(a)<0.02$ and $0.001<\operatorname{Im}(a)<0.1$. This particular choice of the domain allows to avoid numerical difficulties coming from the possible existence of a neutral mode, $a=0$, as well as other instability modes on the coordinate axes. The missing area in the domain $\mathcal{R}$, namely the region $[0,0.001] \times[0,0.1] \cup[0.001,0.02] \times[0,0.001]$, is rather small when compared with the domain $\mathcal{R}$ and can be studied separately.

All the results presented here about the linear stability problem are in dimensionless form. The numerical simulations have been performed assuming the following data for what concerns the kinetic and thermodynamical input parameters as well as the initial 


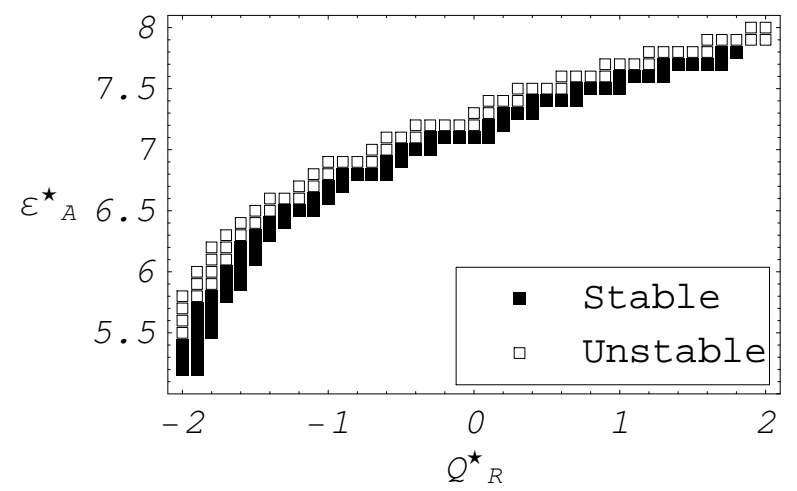

Figure 6. Stability boundary in the $Q_{R}^{*}-\varepsilon_{A}^{\star}$ plane, for the considered region $\mathcal{R}$.

state of the fresh mixture,

$$
\begin{aligned}
& D=1700 \mathrm{~ms}^{-1}, \quad E_{A}=2400 \mathrm{~K}, \quad m=0.01 \mathrm{Kg} / \mathrm{mol}, \\
& n_{A}^{+}=0.35 \mathrm{~mol} / \mathrm{l}, \quad n_{B}^{+}=0 \mathrm{~mol} / \mathrm{l}, \quad T^{+}=298.15 \mathrm{~K} .
\end{aligned}
$$

The considered detonation velocity corresponds to an overdriven detonation. The reaction heat is varying in the range $-2 \leq Q_{R}^{*} \leq 2$, allowing to investigate the stability for both types of exothermic and endothermic chemical reactions. Furthermore, the equilibrium final state at the end of the reaction zone is assumed to be that point where the derivative of the number density of the constituent $A$ reaches the value $10^{-6}$. Figure 6 shows the stability boundary in the parameter plane defined by the reaction heat $Q_{R}^{*}$ and forward activation energy $\varepsilon_{A}^{\star}$, for the considered domain $\mathcal{R}$ in the complex plane, and for the selected detonation velocity. The meaning of this representation is the following: a pair $\left(Q_{R}^{*}, \varepsilon_{A}^{\star}\right)$ in the stability zone indicates that for the corresponding values of the reaction heat and activation energy, no instability modes have been found in the domain $\mathcal{R}$; analogously, a pair $\left(Q_{R}^{*}, \varepsilon_{A}^{\star}\right)$ in the instability zone indicates that for the corresponding values of the reaction heat and activation energy, one instability mode, at least, has been found in the domain $\mathcal{R}$. Moreover, figure 6 reveals that for a fixed value of the activation energy, the detonation becomes stable for larger values of the reaction heat, whereas for a fixed value of the reaction heat, the detonation becomes stable for smaller values of the activation energy. These results are consistent with previous experimental works and numerical simulations, which show that increasing the reaction heat, or decreasing the activation energy, tends to stabilize the detonation. See, for example, the book [23] by J. H. S. Lee and the references therein cited.

A further and detailed analysis can provide a more deep description of the instability spectrum. In particular, if one sets the forward activation energy equal to a fixed value, namely $\varepsilon_{A}^{\star}=7$, and left the reaction heat $Q_{R}^{*}$ varying in a certain range as the parameter of interest, the numerical method allows to count the instability modes. Table 2 shows the number of instability modes that have been found in the domain $\mathcal{R}$, for different values of the reaction heat in the range $-2 \leq Q_{R}^{*} \leq 2$. One can see that the number of instability modes in the region $\mathcal{R}$ is zero when $Q_{R}^{*} \geq-0.6$ and increases for lower values of $Q_{R}^{*}$. These results are in agreement with the behaviour recognizable in figure 
6 as well as with the general trend described above.

Table 2. Number of the instability modes in the domain $\mathcal{R}$, for fixed forward activation energy, $\varepsilon_{A}^{\star}=7$, and different values of the reaction heat in the range $-2 \leq Q_{R}^{*} \leq 2$.

\begin{tabular}{ll}
\hline$Q_{R}^{*}$ & number of modes \\
\hline 2 & 0 \\
1.5 & 0 \\
1 & 0 \\
0 & 0 \\
-0.5 & 0 \\
-0.6 & 0 \\
\hline
\end{tabular}

\begin{tabular}{ll}
\hline$Q_{R}^{*}$ & number of modes \\
\hline-0.62 & 1 \\
-0.65 & 2 to 3 \\
-0.7 & 4 to 7 \\
-1 & 18 to 24 \\
-1.5 & 57 to 70 \\
-2 & 215 to 252 \\
\hline
\end{tabular}

Table 2 suggests the idea that for the considered value of the forward activation energy, $\varepsilon_{A}^{\star}=7$, the number of instability modes increases indefinitely when the reaction heat decreases. Similar results have been obtained in some previous works [37].

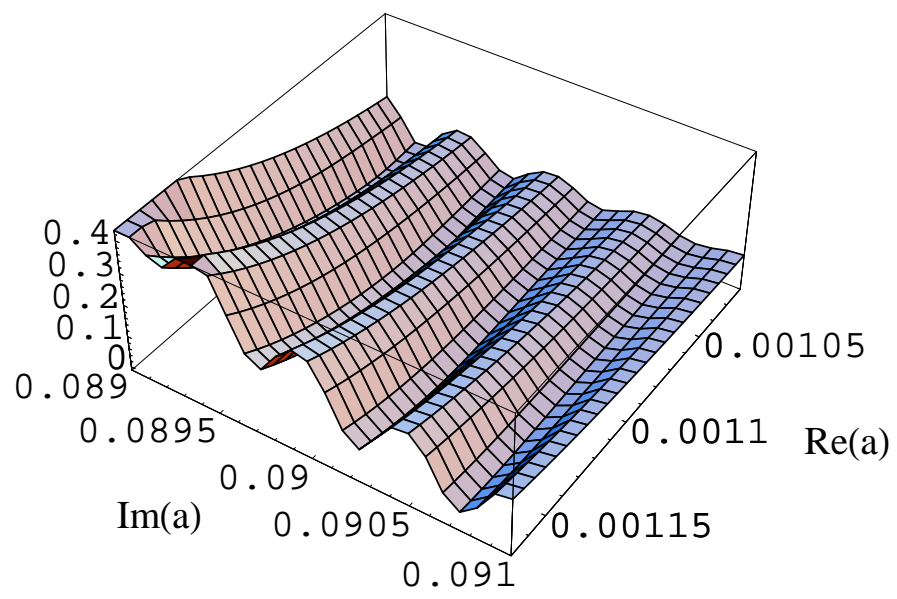

Figure 7. Three-dimensional plot of the magnitude of the residual function, $|\mathscr{H}(a)|$, in the sub-domain of $\mathcal{R}$ defined by $\operatorname{Re}(a) \in[0.00102,0.00117], \operatorname{Im}(a) \in[0.089,0.091]$, for reaction heat and forward activation energy given by $Q_{R}=-0.1$ and $\varepsilon_{A}^{\star}=7.5$.

Figure 7 shows a three-dimensional plot of $|\mathscr{H}(a)|$, for an exothermic chemical reaction with $Q_{R}=-0.1$ and forward activation energy $\varepsilon_{A}^{\star}=7.5$. This plot was obtained with increased resolution in a refinement of the region $\mathcal{R}$, namely in the subregion $[0.00102,0.00117] \times[0.089,0.091]$. A very thin uniform grid is used, with step $10^{-4}$ for the imaginary part and $10^{-5}$ for the real part. The points $a_{j}$ of this grid are assumed as trial values to evaluate the magnitude of the residual function and the instability modes are obtained as the zeros of $|\mathscr{H}(a)|$. Figure 7 shows the existence of four instability modes. A thinner grid should produce accurate approximations for these modes, however the computational effort should become rather intensive. Applying this 


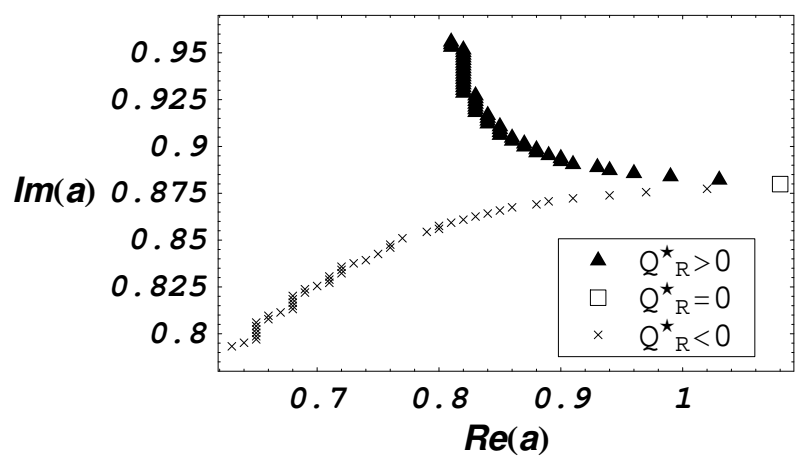

Figure 8. Migration of the fundamental instability mode (lowest perturbation frequency) for the activation energy $\varepsilon_{A}^{\star}=7.5$, as the reaction heat $Q_{R}^{*}$ is varied, with a step of $10^{-2}$, from -0.5 to 0.5 . Re $a$ is scaled by the factor $10^{3}$ and $\operatorname{Im} a$ by the factor 10 .

procedure with grids occupying different regions, one obtains the instability spectra represented bellow, in figure 9 , for different values of the reaction heat $Q_{R}^{*}$. In particular, the spectrum of figure 9 , for $Q_{R}^{*}=-0.1$, includes the four instability modes of figure 7 . Another study concerning the effect of the reaction heat on the stability behaviour is represented in figures 8 and 9 . Figure 8 shows the migration of the fundamental instability mode, corresponding to the lowest perturbation frequency (small imaginary part), for the activation energy $\varepsilon_{A}^{\star}=7.5$, as the reaction heat $Q_{R}^{*}$ is varied from -0.5 to 0.5 . This choice for the range of the reaction heat allows to follow the migration of the fundamental mode when it passes, in particular, through the inert system characterized by the vanishing of the reaction heat, $Q_{R}^{*}=0$. Note that the plot range of figure 8 is not contained in the domain $\mathcal{R}$, already defined, but they intercept each other. In figure 8 , the inert system is represented by the square labeled point, which is located on the right-hand-side of the frame. All the points labeled with the cross correspond to $Q_{R}^{*}<0$, or ZND detonation with exothermic chemical reaction, whereas the points labeled with the black triangle correspond to $Q_{R}^{*}>0$, or pathological stage of the detonation with endothermic chemical reaction. The mode departs from the crossed point located on the left-hand-side of the frame, corresponding to $Q_{R}^{*}=-0.5$, and starts moving above and to the right, until $Q_{R}^{*}$ reaches its zero value at the square labeled point. This trend means that the perturbation frequency increases and the growth rate also increases, so that a destabilizing effect of the detonation is verified. Then, when $Q_{R}^{*}$ increases from its zero value to positive values, the mode moves above and to the left. This behaviour means that the perturbation frequency increases but the growth rate decreases, so that a stabilizing effect of the detonation is observed. Therefore one can conclude that the endothermic reaction $\left(Q_{R}^{*}>0\right)$ has a stabilizing effect on the detonation wave. The results shown in figure 8 are in agreement with those provided in other previous works on detonation stability, see for example [17, 37, 40, 47].

The unstable spectra in the domain $\mathcal{R}$ are represented in figure 9 , when the forward activation energy is $\varepsilon_{A}^{\star}=7.5$, and the reaction heat takes the values $Q_{R}^{*}=-0.1$, 


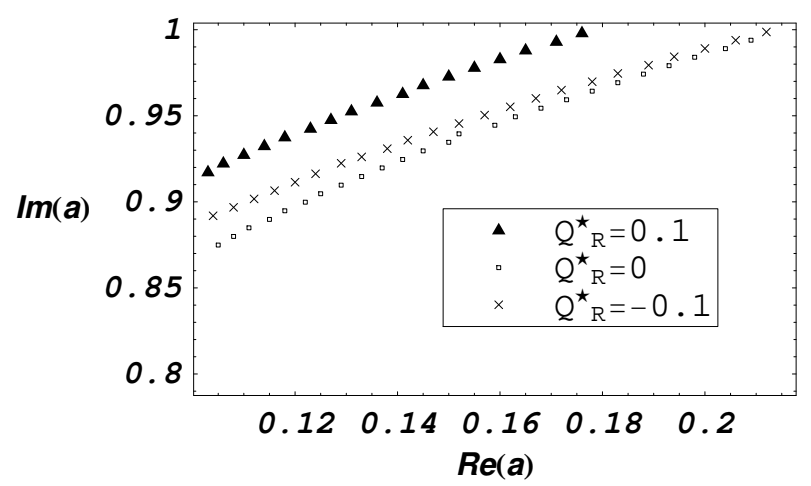

Figure 9. Unstable spectra for the activation energy $\varepsilon_{A}^{\star}=7.5$, as the reaction heat takes the values $Q_{R}^{*}=-0.1, Q_{R}^{*}=0, Q_{R}^{*}=0.1$. Re $a$ is scaled by the factor $10^{2}$ and $\operatorname{Im} a$ by the factor 10 . Zoom at an upper-left sub-domain of $\mathcal{R}$.

$Q_{R}^{*}=0, Q_{R}^{*}=0.1$. All the instability modes in the domain $\mathcal{R}$ are located in the upper-left sub-domain $[0.1,0.22] \times[0.79,1]$ considered in figure 9 . These modes were obtained using various three-dimensional plots of $|\mathscr{H}(a)|$ similar to the one drawn in figure 7 . Each curve of figure 9 consists of all instability modes that have been found in the considered searching window, for the corresponding value of the reaction heat. In particular, the four instability modes with lower perturbation frequency shown in the instability spectrum for $Q_{R}^{*}=-0.1$ are those modes previously represented in the threedimensional plot of figure 7. Similarly, the instability mode with the lowest perturbation frequency shown in the instability spectrum for $Q_{R}^{*}=0$ is the one represented in figure 8 at the square labeled point. Since the lower curve corresponds to the inert case $\left(Q_{R}^{*}=0\right)$ and all the instability modes for both positive and negative reaction heat are located in the area above this curve, figure 9 suggests the idea that all other possible instability modes are located above the inert curve for $Q_{R}^{*}=0$, and thus the region below the curve corresponds to a stability region. Another interesting feature of figure 9 is the following. Since the perturbation frequency increases with the growth rate, this figure suggests that, for a fixed growth rate, the perturbation frequency of the inert instability mode may be seen as the lower bound of the instability perturbation frequencies; conversely, for a fixed perturbation frequency, the growth rate of the inert instability mode may be seen as the upper bound of the instability perturbation frequencies.

\section{Conclusion and final remarks}

In the present paper, the propagation of the steady detonation wave is studied, resorting to the Zeldovich, von-Neuman and Doering idealized model of detonation. The onedimensional linear stability of the steady detonation wave is then investigated, with emphasis on the influence of the reaction heat on the instability behaviour. The study of both the propagation and linear stability is based on the reactive Euler equations derived from the Boltzmann equations and closure procedure proposed in paper [6] for 
a binary mixture with reversible reaction of symmetric type. Assuming a chemical regime of slow reactive process, the Euler equations obtained with the kinetic approach incorporate explicit contributions of the reaction heat and activation energy, which result to be fundamental for the stability analysis. In fact, this explicit dependence permits to obtain a rather complete analysis, in comparison to the previous investigations based on a kinetic approach.

The numerical method of solution proposed in this paper for the stability problem combines the original ideas posed by Erpenbeck [33] and the sooting technique proposed by Lee and Stewart [37]. Some representative results are given concerning both the steady detonation solution of ZND and pathological type, and the related linear stability spectra. whole structure of the ZND solution with exothermic chemical reaction, as well as the second branch of the pathological solution with endothermic chemical reaction. On the other hand, concerning the hydrodynamic linear stability, this paper presents the first exhaustive study based on a kinetic theory for chemically reactive mixtures. The numerical results show that the stability of the detonation wave is attained for increasing the reaction heat or decreasing the activation energy. Moreover, the stability boundary in the parameter plane $Q_{R}^{*}-\varepsilon_{A}^{*}$, the migration of the fundamental instability mode with lowest perturbation frequency and the unstable spectra are given, showing a good agreement with the analytical and numerical predictions known from the classical detonation literature.

Some prospects of the present linear stability approach are the following. First, the present study can be easily extended to a quaternary reactive mixture, starting from the kinetic modelling and macroscopic closure procedure referred to a mixture of four constituents, in order to consider more general detonating mixtures. Then, the application of the present approach to the CJ idealized detonation in a reactive mixture of two or four constituents should not be a difficult task. Another interesting extension of the present study should be the analysis of the complete structure and stability of the pathological detonation, by considering two consecutive chemical reactions, the former being of exothermic type describing the branch between the shock front and the pathological point and the latter being of endothermic type describing the branch between the pathological point and the final equilibrium state. Finally, the numerical method here proposed should be improved and the hydrodynamic instability of the detonation wave with bi-dimensional disturbances can be analyzed.

\section{Acknowledgments}

This research was partially supported by the FCT Phd Grant SFRH/BD/28795/2006, by the Research Centre of Mathematics of the University of Minho through the FEDER Funds "Programa Operacional Factores de Competitividade COMPETE", and by the Portuguese Funds through FCT - "Fundação para a Ciência e Tecnologia" within the Project Est-C/MAT/UI0013/2011. 


\section{References}

[1] Lemarchand A and Nowakowski B 1998 Perturbation of local equilibrium by a chemical wave front J. Chem. Phys. 109, 7028-7037

[2] Giovangigli V 1999 Multicomponent Flow Modeling (Boston: Birkhäuser)

[3] Cukrowski A S 2000 The role of products and a reverse reaction in analysis of nonequilibrium effects in a bimolecular chemical reaction in a dilute gas Physica A 275 134-151

[4] Conforto F, Monaco R, Schürrer F and Ziegler I 2003 Steady detonation waves via the Boltzmann equation for a reacting mixture J. Phys. A: Math. Gen. 36 5381-5398

[5] Shizgal B and Chikhaoui A 2006 On the use temperature parameterized rate coefficients in the estimation of non-equilibrium reaction rates Physica A 365, 317-362

[6] Kremer G M and Soares A J 2007 Effect of reaction heat on Maxwellian distribution functions and rate of reactions J. Stat. Mech. P12003 1-16

[7] Groppi M, Aoki K, Spiga G and Tritsch V 2008 Shock structure analysis in chemically reacting gas mixtures by a relaxation-time kinetic model Phys. Fluids 20117103 1-11

[8] Kremer G M 2010 An introduction to the Boltzmann equation and transport processes in gases (Berlin: Springer)

[9] Bisi M, Groppi M and Spiga G 2010 KRM Flame structure from a kinetic model for chemical reactions 3 17-34

[10] Pandolfi Bianchi M and Soares A J 2011 Kinetic modelling and solutions to the linear stability of a detonation wave J. Difference Eqs. Applic. 17 1169-1184

[11] Lemarchand A, Nowakowski B, Dumazer G and Antoine C 2011 Microscopic simulations of supersonic and subsonic exothermic chemical wave fronts and transition to detonation, J. Chem. Phys. 134, 034121, 1-11

[12] Conforto, F, Groppi M, Monaco R and Spiga G 2011 KRM Kinetic Approach to deflagration processes in a recombination reaction 4 259-276

[13] Abouseif G E and Toong T Y 1982 Theory of unstable one-dimensional detonations Combustion E Flame 45 67-94

[14] Abouseif G E and Toong T Y 1986 Theory of unstable two-dimensional detonations: Genesis of the transverse waves Combustion \& Flame 63 191-207

[15] Cooper M, Jacksony S, Austin J, Wintenberger E and Shepherdz J E 2002 J. Propulsion and Power Direct experimental impulse measurements for detonations and deflagrations 18 1033-1041

[16] Pintgena F, Ecketta C A, Austin J M and Shepher J E 2003 Combustion \& Flame Direct observations of reaction zone structure in propagating detonations 133 211-229

[17] Short M 2005 Theory and modeling of detonation wave stability: a brief look at the past and toward the future 20th Int. Colloq. Dyn. Expl. React. Syst., 1-19

[18] Stewart D S and Kasimov A 2006 State of Detonation Stability Theory and Its Application to Propulsion J. Propulsion and Power 22, 1230-1244

[19] Carvalho F and Soares A J 2010 Steady detonation wave solutions under the reaction heat effect' Proc. XV International Conference WASCOM (Singapore: World Scientific) p 87-92

[20] Carvalho F and Soares A J 2011 Influence of the reaction heat on the linear stability spectra of steady detonation in the kinetic frame AIP Conf. Proc. of the 27th International Symposium on Rarefied Gas Dynamics (American Institute of Physics) vol 1333 p 69-74

[21] Fickett W and Davis W C 1979 Detonation, Theory and Experiment (Berkeley: University of California)

[22] Fickett W 1986 Introduction to Detonation Theory (Berkeley: University of California)

[23] Lee J H S 2008 The Detonation Phenomenon (Cambridge: Cambridge University Press)

[24] Zhang F (Ed.) 2009 Heterogeneous Detonation Series "Shock Wave Science and Technology Reference Library" 4 (Springer-Verlag: Berlin Heidelberg)

[25] Wood W W and Kirkwood J G 1954 J. Chem. Phys. Structure of a steady state plane detonation wave with finite reaction rate 22 1915-1919 
[26] Wood W W and Kirkwood J G 1954 J. Chem. Phys. Diameter effect in condensed explosives. The relation between velocity and radius of curvature of the detonation wave 22 1920-1924

[27] Torrisi M and Lombardo S 1991 Int. J. Engng. Sci, Similarity solutions of the Wood-Kirkwood model in detonation theory 29 523-532

[28] Aslam Tariq D and Stewart D S 1999 Combust. TheoryModelling Detonation shock dynamics and comparisons with direct numerical simulation 3 77-101

[29] Sharpe G J 1999 Linear stability of pathological detonations J. Fluid Mech. 401 311-338

[30] Sharpe G J and Falle S A E G 2000 One-dimensional nonlinear stability of pathological detonations J. Fluid Mech. 414, 339-366

[31] Sharpe G J and Falle S A E G 2000 Numerical simulations of pulsating detonations: I. Nonlinear stability os steady detonations Combust. Theory Modelling 4, 557-574

[32] Erpenbeck J J 1962 Stability of steady-state equilibrium detonations Phys. Fluids 5 604-614

[33] Erpenbeck J J 1964 Stability of idealized one-reaction detonations Phys. Fluids 7 684-696

[34] Buckmaster J D and Ludford G S S 1986 The effect of structure on the stability of detonations I. role of the induction zone Proc. XX Simp. Comb. 1669-1676

[35] Buckmaster J D and Neves J 1988 One-dimensional detonation stability: The spectrum for infinite activation energy Phys. Fluids 31 3571-3576

[36] Majda A J and Rosales R 1983 A Theory for Spontaneous Mach Stem Formation in Reacting Shock Fronts, I. The Basic Perturbation Analysis SIAM J. Appl. Math. 43 1310-1334

[37] Lee H I and Stewart D S 1990 Calculation of linear detonation stability: one dimensional instability of plane detonation J. Fluid Mech. 216, 103-132

[38] Bourlioux A, Majda A and Roytburd V 1991 Theoretical and numerical structure for unstable one-dimensional detonations SIAM J. Appl. Math 51 303-343

[39] He L and Lee J H S 1995 The dynamical limit of one-dimensional detonations Phys. Fluids 7 $1151-1158$

[40] Sharpe G J 1997 Linear stability of idealized detonations Proc. R. Soc. Lond. A 2623-2605

[41] Short M and Stewart D S 1998 Cellular detonation stability. Part 1. A normal-mode linear analysis J. Fluid Mech. 368, 229-262

[42] Kasimov A and Stewart D S 2002 Spinning instability of gaseous detonations J. Fluid Mech. 466 179-203

[43] Papalexandris Miltiadis V 2004 Numerical simulation of detonations in mixtures of gases and solid particles J. Fluid Mech. 507 95-142

[44] Mazaheri K, Hashemi S S and Lee J H 2004 Numerical study of detonation instability for a two-step kinetics model Scientia Iranica 11 292-301

[45] Liang Z, Khastoo B, and Bauwens L 2004 Effect of reaction order on stability of planar detonation Int. J. Comput. Fluid Dyn. 19 131-142

[46] Kasimov A and Stewart D S 2005 Theory of detonation with an embedded sonic locus SIAM J. Appl. Math. 66, 384-407

[47] Gorchkov V, Kiyanda C B, Short M and Quirk J J, 2007 A detonation stability formulation for arbitrary equations of state and multi-step reaction mechanisms Proc. Combust. Instit 31 $2397 ? 2405$

[48] Sharpe G J and Falle S A E G 1999 One-dimensional numerical simulations of idealized detonations Proc. R. Soc. Lond. A 455 1203-1214

[49] Chevalier J M, Carion N, Protat J C and Redasse J C 1993 Propagation phenomena on the detonation wave front Phys. Rev. Lett. 71 712-715

[50] Austina J M, Pintgenb F and Shepherd J E 2005 Reaction zones in highly unstable detonations Proc. Comb. Inst. 30 1849-1857

[51] Watt S D and Sharpe G J 2005 Linear and nonlinear dynamics of cylindrically and spherically expanding detonation waves J. Fluid Mech. 522, 329-356 